\title{
Ajustamento Conjugal: Comparação entre Casais com e sem Filhos com Deficiência Intelectual
}

\author{
Nara Liana Pereira-Silva - Universidade Federal de Juiz de Fora, Juiz de Fora, Brasil \\ Maria Auxiliadora Dessen - Universidade de Brasilia, Brasilia, Brasil \\ Altemir José Gonçalves Barbosa - Universidade Federal de Juiz de Fora, Juiz de Fora, Brasil
}

\begin{abstract}
Resumo
O presente estudo visa comparar o ajustamento diádico entre casais com um filho com deficiência intelectual e casais com filhos com desenvolvimento típico, identificando as associações dessa variável com características do funcionamento do sistema familiar. Participaram do estudo 101 famílias, sendo 80 com filhos com desenvolvimento típico e 21 com um dos filhos com deficiência intelectual. Os instrumentos utilizados foram: Questionário de Caracterização do Sistema Familiar e a Escala de Ajustamento Diádico - EAD (DAS - Dyadic Adjustment Scale). Os resultados mostram que o relacionamento conjugal, em ambos os grupos, é bom e não há diferenças significativas quanto ao ajustamento diádico entre os casais. A análise das dimensões da EAD mostra correlação significativa, positiva e moderada, apenas para Expressão de Afeto. Os dados indicam que a deficiência do filho não constitui empecilho ao ajustamento diádico do casal, fato que deveria ser melhor investigado em famílias brasileiras. Palavras-chave: Família, ajustamento diádico, conflito conjugal, deficiência intelectual, desenvolvimento típico.
\end{abstract}

\section{Marital Adjustment: Comparison between Couples with Children with and without Intellectual Disability}

\begin{abstract}
This study aims to compare the dyadic adjustment between couples with a child with intellectual disabilities and couples with children with typical development identifying the associations of this variable with characteristics of the functioning of the family system. The study included 101 families, 80 of them with children with typical development and 21 of them with one of the children with intellectual disabilities. The instruments used were Family Characteristics Questionnaire and Dyadic Adjustment Scale - DAS. The results show that the marital relationship, in both groups, is good and there are no significant differences regarding the dyadic adjustment between couples. The analysis of the dimensions of the DAS shows significant correlation, positive and moderate, but only in relation to the variable Affect Expression. The data indicate that the child's disability is not a hindrance to the couple dyadic adjustment, a fact that should be further investigated in Brazilian families.

Keywords: Family, dyadic adjustment, marital conflict, intellectual disability, typical development.
\end{abstract}

\section{Ajuste Conyugal: Comparación entre Parejas con y sin Hijos con Discapacidad Intelectual}

\begin{abstract}
Resumen
El presente estudio objetiva comparar el ajuste conyugal entre parejas con un hijo con discapacidad intelectual y parejas con hijos con desarrollo típico, identificando las asociaciones de esta variable con las características del funcionamiento del sistema familiar. Participaron 101 familias, siendo 80 de ellas con hijos con desarrollo típico y 21 con uno de los hijos con discapacidad intelectual. Se utilizaron el Cuestionario de Caracterización del Sistema Familiar y la Escala de Ajuste Conyugal - EAC (DAS-Dyadic Adjustment Scale). Los resultados muestran que la relación conyugal, en ambos grupos, es buena y no hay diferencias significativas en el ajuste conyugal entre las parejas. El análisis de las dimensiones de la EAC muestra correlación significativa, positiva y moderada, solamente para la Expresión de Afecto. Los datos indican que la discapacidad del hijo no constituye un obstáculo para el ajuste conyugal de la pareja, hecho que debería ser mejor investigado en las familias brasileñas.

Palabras clave: Familia, ajuste conyugal, conflicto conyugal, discapacidad intelectual, desarrollo típico.
\end{abstract}

\section{Introdução}

A qualidade do relacionamento do casal vem sendo estudada desde a primeira metade do século XX. O primeiro estudo sobre ajustamento conjugal é datado de 1929 (Spanier, 1976) e, em 1938, foi realizada a primeira investigação sobre satisfação marital (Heyman, Sayers, \& Bellack, 1994). Entretanto, foi somente a partir de meados da década de 80 que os pesquisadores passaram a considerar as dimensões do relacionamento conjugal como variável fundamental para a compreensão das relações familiares, particularmente das relações parentais (Dessen \& Braz, 2005). Sabe-se, hoje, que as relações conjugais afetam diretamente as relações que o casal mantém com seus filhos, assim como as relações parentais afetam a dinâmica de relacionamento do casal, ocorrendo um entrejogo de influências mútuas (Braz, Dessen, \& Pereira-Silva, 2005; Erel \& Burman, 1995; Kreppner, 2000; Villas-Boas, Dessen, \& Melchiori, 2010).

Por se tratar de um campo de investigação em expansão, os resultados das pesquisas da década de 1990 sobre o relacionamento de casais que tinham filho com deficiência intelectual refletiam as controvérsias 
existentes nessa área de pesquisa. Dois estudos do final da década de 1980, o de Carr (1988) e o de Bristol, Gallagher e Schopler (1988), não encontraram diferenças significativas entre as famílias de crianças com e sem deficiência intelectual (DI), especialmente aquelas com síndrome de Down, no tocante à qualidade da relação marital, coesão e satisfação conjugal. No entanto, Shapiro, Blacher e Lopez (1998) identificaram estudos constatando alto índice de divórcio e discórdia marital em genitores (casais) de crianças com síndrome de Down (SD), apesar de terem encontrado, também, estudos que não mostravam diferenças entre os índices de divórcio e discórdia quando se comparavam casais com crianças com e sem DI.

Segundo esses autores (Shapiro, Blacher, \& Lopez, 1998), as famílias de crianças com DI não apresentam, necessariamente, mais disfunções em suas vidas que as familias com crianças com desenvolvimento típico (DT) e, também, não possuem casamentos piores ou sistemas sociais de apoio menos adequados. Segundo Floyd e Zmich (1991), que investigaram 38 famílias de crianças com DI e 34 de crianças com DT, a insatisfação marital estava mais associada às medidas de problemas de comportamento do(a) filho(a) do que às medidas da relação conjugal. Esses autores também verificaram, nesse estudo, que o estresse marital estava mais associado às demandas de cuidado com as necessidades especiais da criança, às interações maritais negativas e à ocorrência de trocas aversivas com a criança.

A qualidade da relação conjugal de genitores em famílias de crianças com DI ou com DT foi objeto de uma metanálise conduzida por Risdal e Singer (2004). Essa metanálise comparou a qualidade conjugal de genitores de crianças com e sem deficiência recuperando estudos com resultados discrepantes; alguns relataram pior qualidade conjugal, outros não encontraram nenhuma diferença na qualidade do relacionamento entre o casal; e outros apontaram níveis superiores de qualidade para casais com uma criança com deficiência. Para superar as controvérsias observadas em décadas anteriores (por exemplo, Risdal \& Singer, 2004; Shapiro et al., 1998) e, em decorrência da adoção da perspectiva sistêmica da família, os estudos passaram a empregar delineamentos caracterizados por um maior rigor metodológico na coleta e na análise dos dados (Dykens, 2005). Os delineamentos longitudinais são recomendados, a definição do grupo a ser pesquisado é extremamente importante, considerando as especificidades de cada tipo de etiologia da deficiência, além da importância em se utilizar a combinação de instrumentos qualitativos e quantitativos.

Como consequência da mudança no modo de pesquisar, os resultados têm sinalizado a existência de diversos fatores que, inter-relacionados, interferem na qualidade da relação dos casais que têm filhos com DI, tais como problemas de comportamento da criança, bem-estar dos genitores, nível de escolaridade, renda familiar (Norlin \& Broberg, 2012; Stoneman, Gavidia-Payne, \& Floyd 2006; Wieland \& Baker, 2010). Além disso, tem-se observado um panorama mais positivo em relação às famílias com crianças com DI (por exemplo, Bailey Junior, 2007; Davis \& Gavidia-Payne, 2009).

Os estudos sobre a satisfação, o suporte/apoio e o ajustamento conjugal, que representam alguns dos indicadores da qualidade da relação conjugal, têm contribuído, particularmente, para superar as limitações e reduzir as controvérsias a respeito das diferenças entre casais com filhos com DI e casais com filhos com DT. No que tange à satisfação conjugal, cujo conceito é diversificado, complexo e vem sendo associado a diferentes fatores (Mosmann, Wagner, \& Féres-Carneiro, 2006; Perlin \& Diniz, 2005; Scorsolini-Comin \& Santos, 2010; Wagner \& Falcke, 2001), os resultados denotam que, em famílias de crianças com DI, os níveis de satisfação conjugal são considerados bons, isto é, iguais ou superiores à média (Da Silva, 2011; Stoneman et al., 2006). No estudo de Da Silva (2011), os casais que tiveram níveis mais altos de satisfação conjugal apresentaram, também, baixos níveis de estresse relacionados à presença da criança e de sintomas de depressão.

Segundo Bristol et al. (1988), o suporte marital é uma variável de extrema relevância na análise da qualidade da relação entre os casais, com sua influência estendendo-se para a relação genitores-criança. No estudo de Stoneman et al. (2006), com 67 famílias de crianças com DI, os resultados evidenciaram alto ajustamento marital para esposas cujos maridos empregavam mais estratégias de enfrentamento de problemas e, portanto, conseguiam oferecer maior apoio. Essa constatação explicita a importância de se considerar a natureza sistêmica da família, uma vez que os estressores, as estratégias de enfrentamento e o bem-estar marital, dentre outras variáveis, têm influências bidirecionais e atingem os diversos subsistemas familiares.

O ajustamento diádico, por sua vez, foi o conceito mais analisado nas pesquisas sobre conjugalidade até meados da década de 90 (Mossman et al., 2006). De acordo com esse autor, a Escala de Ajustamento Diádico 
(DAS - Dyadic Adjustment Scale) elaborada por Spanier (1976) foi utilizada em mais de 1000 estudos, visando medir as dimensões dessa variável. Povee, Roberts, Bourke e Leonard (2012) investigaram uma amostra de 224 cuidadores primários de pessoas com síndrome de Down, utilizando a versão abreviada da DAS. Esses autores constataram uma média ligeiramente mais elevada de ajustamento e satisfação conjugal nesse grupo, comparada à média do grupo de validação. Segundo os autores, tanto as análises qualitativas quanto as quantitativas convergem e revelam que ter um filho com síndrome de Down não teve impacto nessas famílias ou, quando teve, foi mínimo. Os resultados positivos na vida da família foram, provavelmente, alcançados pelo fato de se aceitar a criança com síndrome de Down, adaptando e modificando a vida familiar para atender às necessidades específicas da criança (Povee et al., 2012). No entanto, os próprios autores destacaram que esse estudo foi realizado com uma amostra australiana, país onde as famílias possuem altos níveis de apoio e tem sido relatada grande satisfação com os serviços prestados a elas.

Richardson (2012), ao investigar o relacionamento conjugal em famílias de adolescentes e adultos com deficiência intelectual utilizando a DAS, verificou um aumento dos escores da medida de qualidade conjugal para ambos os genitores, quando os filhos estavam mais próximos da vida adulta. De acordo com a autora, o padrão de relacionamento conjugal encontrado nas familias com filhos com DI é similar ao encontrado em famílias com filhos com DT, em que há um declínio na qualidade do relacionamento conjugal quando os filhos estão na adolescência e, posteriormente, um aumento nos níveis dessa qualidade quando os filhos estão na idade adulta.

O estudo longitudinal de Wieland e Baker (2010), com 132 famílias, das quais 48 possuíam uma criança com DI e 84 possuíam apenas crianças com DT, revelou não haver diferenças significativas na média da qualidade conjugal e nos escores de apoio do cônjuge entre os dois grupos investigados. A variância nos escores de qualidade conjugal foi significativamente maior para os casais com filho com DI do que para aqueles com filho com DT. Além disso, os resultados evidenciaram que escores mais altos no domínio marital estavam relacionados com escores mais baixos de problemas de comportamento da criança. Norlin e Broberg (2012) chegaram a conclusões similares às de Wieland e Baker ao pesquisarem uma amostra composta por crianças com e sem DI associada a problemas de comportamento. Em seu estudo, a qualidade da relação conjugal não diferiu entre os grupos, por outro lado, as análises revelaram diferenças no tocante à coparentalidade. Esses resultados refletem a importância relativa do sistema de coparentalidade em situações de estresse relacionadas com a criança. Segundo os autores, é provável que os desafios relacionados ao crescimento de uma criança com necessidades especiais e problemas de comportamento fortaleçam a capacidade dos genitores para cooperar e apoiar um ao outro.

Tendo em vista a necessidade de compreender melhor o funcionamento de famílias de crianças que apresentam algum tipo de deficiência, particularmente o relacionamento do casal, e, considerando a escassez de pesquisas sobre o tema no Brasil, este estudo visa comparar o ajustamento diádico entre casais com pelo menos um filho com DI e casais com filhos com DT, relacionando-o a características do funcionamento do sistema familiar. Foram destacadas as seguintes dimensões das relações conjugais e do funcionamento familiar para análise: o Consenso, a Satisfação, a Coesão Conjugal e a Expressão de Afeto, bem como a renda familiar, o número de filhos, a divisão de tarefas domésticas e o apoio do parceiro.

\section{Método}

\section{Participantes}

Participaram do estudo 104 díades recrutadas no Distrito Federal que tinham, pelo menos, um filho entre três a sete anos de idade $(\overline{\mathrm{X}}=4,49 ; D P=1,63)$, sendo $53,85 \%(n=56)$ deles do sexo masculino; $43,27 \%(n$ $=45)$ do sexo feminino e $2,88 \%(n=3)$ não tiveram o sexo informado pelos pais. Os casais, casados legalmente ou não, viviam juntos, em média, há 9,6 anos (casais com filhos com DT) e 10,57 anos (casais com filho com DI).

A Tabela 1 mostra que os dois grupos, casais com filho com DI e casais com filho com DT, são homogêneos quanto ao sexo e à idade do filho, à idade do pai e da mãe e aos anos de convivência do casal. Porém, constatou-se que as famílias com filhos com DI possuíam renda menor e mais filhos, verificando-se, portanto, diferenças significativas em relação aos tipos de famílias no tocante a essas duas variáveis.

Por ocasião da coleta de dados, a idade média, em anos, das mães era 33,01 $(D P=7,49)$ e a dos pais 36,55 $(D P=8,40)$. A renda familiar média era de 5,88 $(D P=7,10)$ salários mínimos, variando entre oito centésimos de um salário mínimo a 30 salários mínimos. 
Tabela 1

Características Demográficas das Familias com Filho com Desenvolvimento Típico (DT) e com Deficiência Intelectual (DI)

\begin{tabular}{lccccc}
\hline & \multicolumn{5}{c}{ Grupos } \\
\cline { 2 - 5 } Variáveis & \multicolumn{2}{c}{$\begin{array}{c}\text { Desenvolvimento típico } \\
\text { (DT) }\end{array}$} & $\begin{array}{c}\text { Deficiência intelectual } \\
\text { (DI) }\end{array}$ & \\
\hline Sexo da criança & & & $\chi_{0}^{2}=0,66$ \\
Masculino & 46 & $57,50 \%$ & 10 & $47,62 \%$ & \\
Feminino & 34 & $42,50 \%$ & 11 & $52,38 \%$ & DP \\
\hline & $\mathrm{M}$ & $\mathrm{DP}$ & $\mathrm{M}$ & $\mathrm{U}_{\text {o }}$ \\
Idade da criança & 4,51 & 1,17 & 4,43 & 2,73 & 861,50 \\
Idade do pai & 36,94 & 8,53 & 35,14 & 7,91 & 814,00 \\
Idade da mãe & 33,47 & 7,15 & 31,39 & 8,53 & 786,50 \\
Anos de convivência do casal & 9,61 & 4,75 & 10,57 & 7,49 & 828,50 \\
Número de filhos & 1,93 & 1,18 & 3,09 & 1,44 & $501,50^{*}$ \\
Renda familiar & 6,72 & 7,40 & 2,77 & 4,77 & $573,00^{*}$ \\
\hline
\end{tabular}

${ }^{\mathrm{a}} N=101$; três díades não informaram o sexo da criança. $g l=1 .{ }^{\mathrm{b}}$ Salários mínimos. ${ }^{*} p<0,05$.

A média de filhos das díades era 2,18 $(D P=1,33)$, variando de um a seis, assim distribuídos: um $(n=48$; $46,15 \%)$, dois $(n=13 ; 12,50 \%)$, três $(n=27 ; 25,96$ $\%)$, quatro $(n=11 ; 10,58 \%)$, cinco $(n=2 ; 1,92 \%) \mathrm{e}$ seis $(n=3 ; 2,88)$. As díades foram divididas em dois grupos: aquelas somente com filhos(as) com DT $(n=$ $81 ; 77,88 \%$ ) e aquelas com um dos filhos(as) com DI $(n=23 ; 22,12 \%)$.

As crianças com deficiência intelectual tinham o diagnóstico de síndrome de Down $(n=12 ; 52,17 \%)$, de paralisia cerebral com comprometimento cognitivo $(n=2 ; 8,70 \%)$, microcefalia $(n=1 ; 4,35 \%)$, síndrome de Klinefelter $(n=1 ; 4,35 \%)$, síndrome de Rubinstein-Taybi $(n=1 ; 4,35 \%)$, DI sem especificação $(n=1$; $4,35 \%)$, encefalite $(n=1 ; 4,35 \%)$, encefalocele $(n=1$; $4,35 \%)$ ou toxoplasmose fetal $(n=1 ; 4,35 \%)$. Duas díades $(8,70 \%)$ não informaram o diagnóstico exato do filho, mencionando apenas DI.

\section{Instrumentos}

Foram utilizados, para a coleta de dados, o Questionário de Caracterização do Sistema Familiar (Dessen, 2009) e a DAS - Dyadic Adjustment Scale (Spanier, 1976) em seu formato traduzido para a língua portuguesa do Brasil (Escala de Ajustamento Diádico - EAD). O Questionário de Caracterização do Sistema Familiar é um instrumento que tem o objetivo, não só de coletar informações sociodemográficas, tais como idade e escolaridade dos genitores e filhos, renda familiar, ocupação dos genitores, estado civil, contatos sociais da familia, mas também dados sobre a rede social de apoio, a divisão de tarefas rotineiras desenvolvidas com a criança, atividades de lazer da família, dentre outros dados do contexto familiar.

A EAD avalia quatro dimensões do ajustamento conjugal, por meio de 32 itens, fazendo uso de escala tipo Likert com possibilidades de resposta de 5, 6 ou 7 pontos, tendo dois itens (29 e 30) com apenas duas opções ("sim" ou "não"). A primeira dimensão - Consenso Diádico, refere-se à percepção individual de aspectos do relacionamento e do nível de concordância do casal sobre uma variedade de questões básicas, tais como financeiras, atividades de lazer, religiosas, amizades, de convencionalidade, de filosofia de vida, de negócios com parentes, de metas e objetivos, de tempo alocado, de participação na tomada de decisão, de participação nas tarefas domésticas, de decisões sobre as carreiras profissionais. A Satisfação Diádica examina as percepções de cada um a respeito de situações que envolvem a possibilidade do divórcio e/ou separação, da saída de casa, do arrependimento, do bem-estar, da confiança, da frequência de emissão de beijo, da questão da felicidade e do compromisso com o relacionamento. Já a Coesão Diádica mede o grau de compartilhamento emocional do casal, bem como as percepções individuais relativas ao engajamento mútuo em interesses externos, à criação de ideias, à diversão conjunta, à discussão tranquila e ao trabalho conjunto em projetos. A Expressão de Afeto entre o casal é a quarta dimensão avaliada pela EAD. 
A pontuação total da EAD é obtida a partir da soma dos pontos de todos os itens, variando entre $0 \mathrm{e}$ 151. Os escores das dimensões da escala são obtidos somando os pontos dos itens de cada uma delas, ou seja, a pontuação varia entre 0 a 65 para o Consenso Diádico, entre 0 a 50 para a Satisfação Diádica, entre 0 a 24 para a Coesão Diádica e entre 0 a 12 para a Expressão de Afeto.

\section{Procedimentos}

O presente estudo foi aprovado pelo Comitê de Ética da Faculdade de Saúde da Universidade de Brasília - CEP/FS/UnB (nº 144). Após a sua aprovação, deu-se início o procedimento de seleção da amostra. Para a seleção das famílias, foram contatadas escolas públicas de educação infantil e centros de educação especial da cidade de Brasília e regiões administrativas do Distrito Federal. As escolas e centros especiais que deram consentimento para a realização da pesquisa foram visitados, e o primeiro contato com as famílias das crianças que preenchiam os critérios da pesquisa foi efetuado, por meio de carta, solicitando a sua participação na pesquisa. As solicitações foram enviadas aos genitores de todas as crianças matriculadas nas escolas e centros especiais que tinham idades entre três e sete anos e eram filhos biológicos de genitores que coabitavam a mesma residência. Além desses critérios, as crianças que frequentavam o centro de educação especial deveriam possuir documentação comprobatória de diagnóstico de deficiência intelectual, consultada mediante permissão da direção dos respectivos centros.

As famílias que responderam à solicitação, dando o seu consentimento para a participação na pesquisa, foram contatadas por telefone e uma visita à casa foi agendada. Na primeira visita, foi assinado o Termo de Consentimento Livre e Esclarecido e, em seguida, a mãe respondeu às questões do Questionário de Caracterização do Sistema Familiar (Dessen, 2009). Na segunda visita, o pai e a mãe responderam, separadamente, à Escala de Ajustamento Diádico - EAD (Spanier, 1976).

Os dados foram tabulados em uma planilha eletrônica e submetidos a uma análise quantitativa. Além de estatística descritiva (média, porcentagem, etc.), empregaram-se provas inferenciais para comparar os grupos DI e DT. Devido ao tipo de medida - nominal - e à ausência de distribuição normal dos dados, optou-se por utilizar testes não paramétricos (teste $\mathrm{U}$ de Mann Whitney e Qui-Quadrado), com um nível de significância de 5\%. A classificação do ajustamento das díades com base nos escores da EAD foi feita de acordo com os critérios propostos por Spanier (1976). Assim, pontuações iguais ou inferiores a 101 correspondem a díades desajustadas e resultados superiores, a díades ajustadas.

\section{Resultados}

Ao considerar algumas atividades que as famílias realizam com seus filhos (Tabela 2), constatou-se que as díades com crianças com DT diferem estatisticamente das que possuem filhos com DI somente no caso da atividade de ler/contar histórias. Nas famílias com filho com DT, essa atividade é mais frequentemente realizada com a participação conjunta do pai e da mãe, enquanto naquelas com filhos com DI, a mãe desenvolve essa atividade mais frequentemente em conjunto com outras pessoas do que com o pai. Destaca-se que, nas atividades de alimentação e banho, não há participação do pai, considerando ambos os grupos. Essas atividades têm a inserção do pai somente em conjunto com a mãe. Levar o filho à escola é uma atividade realizada mais pelas mães, em ambos os grupos, apesar de se observar que o pai também a realiza, mas com uma frequência menor. Apesar de não se verificar diferenças estatisticamente significantes entre os grupos no que se refere a levar a criança para atividades de lazer, destaca-se que nenhum pai com filho com DI a realiza sozinho, sendo essa a atividade que mais tem a participação conjunta entre o pai e a mãe desse grupo. Colocar o filho para dormir também é uma atividade mais realizada pela mãe e menos pelo pai em famílias com filho com DI. Já em famílias com filho com DT, essa atividade é mais frequentemente realizada de forma conjunta entre o pai e a mãe.

Em se tratando das tarefas domésticas, observou-se não haver diferenças estatísticas entre os grupos, sendo a mãe a principal responsável por essas atividades, realizando-as sozinha ou em conjunto com o parceiro. O pai, em ambos os grupos, não realiza sozinho as atividades de "limpar a casa" e "cozinhar". A tarefa de "comprar comida" apresentou maior frequência do compartilhamento entre o casal em ambos os grupos.

Mãe e pai com filhos com DT compartilham mais a responsabilidade pelas atividades com o filho e com as tarefas domésticas $(M=2,51 ; D P=1,71)$ do que os genitores com filho com DI $(M=1,48 ; D P=1,70)$, teste $\mathrm{U}$ de Mann Whitney $=551,00 *$, quando se trata do total de atividades realizadas. Verificou-se, também, que as mães cujos filhos possuem DI assumem sozinhas ( $M$ 
Tabela 2

Responsáveis Pelas Atividades Realizadas com o Filho e Pelas Tarefas Domésticas, de Acordo com os Dois Grupos (DT e DI)

\begin{tabular}{|c|c|c|c|c|c|c|}
\hline \multirow{3}{*}{ Atividades } & \multirow{3}{*}{ Responsável } & \multicolumn{4}{|c|}{ Grupos } & \multirow{3}{*}{ Phi } \\
\hline & & \multicolumn{2}{|c|}{$\begin{array}{l}\text { Desenvolvimento típico } \\
\text { (DT) }\end{array}$} & \multicolumn{2}{|c|}{$\begin{array}{l}\text { Deficiência intelectual } \\
\text { (DI) }\end{array}$} & \\
\hline & & $\mathrm{n}$ & $\%$ & $\mathrm{n}$ & $\%$ & \\
\hline \multirow{4}{*}{ Alimentação e banho ${ }^{a}$} & Mãe & 24 & 30,77 & 11 & 47,83 & \multirow{4}{*}{0,15} \\
\hline & $\mathrm{Pai}$ & - & - & - & - & \\
\hline & Mãe e pai & 11 & 14,10 & 2 & 8,70 & \\
\hline & Outros & 43 & 55,13 & 10 & 43,48 & \\
\hline \multirow{4}{*}{ Levar à escolab } & Mãe & 24 & 34,29 & 12 & 54,55 & \multirow{4}{*}{0,22} \\
\hline & Pai & 13 & 18,57 & 3 & 13,64 & \\
\hline & Mãe e pai & 15 & 21,43 & 1 & 4,55 & \\
\hline & Outros & 18 & 25,71 & 6 & 27,27 & \\
\hline \multirow{4}{*}{ Ler/contra histórias ${ }^{\mathrm{c}}$} & Mãe & 25 & 35,21 & 7 & 36,84 & \multirow{4}{*}{$0,38 *$} \\
\hline & Pai & 13 & 18,31 & 1 & 5,26 & \\
\hline & Mãe e pai & 25 & 35,21 & 2 & 10,53 & \\
\hline & Outros & 8 & 11,27 & 9 & 47,37 & \\
\hline \multirow{4}{*}{ Levar para atividades de lazer ${ }^{d}$} & Mãe & 12 & 15,79 & 8 & 36,36 & \multirow{4}{*}{0,22} \\
\hline & Pai & 3 & 3,95 & - & - & \\
\hline & Mãe e pai & 46 & 60,53 & 10 & 45,45 & \\
\hline & Outros & 15 & 19,74 & 4 & 18,18 & \\
\hline \multirow{4}{*}{ Colocar o filho para dormir ${ }^{\mathrm{e}}$} & Mãe & 28 & 36,36 & 12 & 52,17 & \multirow{4}{*}{0,19} \\
\hline & Pai & 10 & 12,99 & 4 & 17,39 & \\
\hline & Mãe e pai & 30 & 38,96 & 4 & 17,39 & \\
\hline & Outros & 9 & 11,69 & 3 & 13,04 & \\
\hline \multirow{4}{*}{ Limpar a casa ${ }^{a}$} & Mãe & 21 & 26,92 & 11 & 47,83 & \multirow{4}{*}{0,19} \\
\hline & Pai & - & - & - & - & \\
\hline & Mãe e pai & 9 & 11,54 & 1 & 4,35 & \\
\hline & Outros & 48 & 61,54 & 11 & 47,83 & \\
\hline \multirow{4}{*}{ Cozinhar ${ }^{\mathrm{e}}$} & Mãe & 24 & 31,17 & 12 & 52,17 & \multirow{4}{*}{0,18} \\
\hline & Pai & - & - & - & - & \\
\hline & Mãe e pai & 11 & 14,29 & 2 & 8,70 & \\
\hline & Outros & 42 & 54,55 & 9 & 39,13 & \\
\hline \multirow{4}{*}{ Lavar e passar roupas ${ }^{a}$} & Mãe & 25 & 32,05 & 13 & 56,52 & \multirow{4}{*}{0,22} \\
\hline & Pai & 1 & 1,28 & - & - & \\
\hline & Mãe e pai & 2 & 2,56 & 1 & 4,35 & \\
\hline & Outros & 50 & 64,10 & 9 & 39,13 & \\
\hline \multirow{4}{*}{ Comprar comida ${ }^{a}$} & Mãe & 21 & 26,92 & 6 & 26,09 & \multirow{4}{*}{0,09} \\
\hline & Pai & 11 & 14,10 & 5 & 21,74 & \\
\hline & Mãe e pai & 40 & 51,28 & 10 & 43,48 & \\
\hline & Outros & 6 & 7,69 & 2 & 8,70 & \\
\hline & Mãe & 36 & 76,60 & 6 & 75,00 & \\
\hline Orientar a empregada nas & Pai & 1 & 2,13 & - & - & 74 \\
\hline tarefas domésticas $^{\mathrm{f}}$ & Mãe e pai & 7 & 14,89 & 1 & 12,50 & $0, / 4$ \\
\hline & Outros & 3 & 6,38 & 1 & 12,50 & \\
\hline
\end{tabular}

${ }^{\mathrm{a}} N=101 ;{ }^{\mathrm{b}} N=92 ;{ }^{\mathrm{c}} N=90 ;{ }^{\mathrm{d}} N=98 ;{ }^{\mathrm{e}} N=100 ;{ }^{\mathrm{f}} N=55 . * p<0,05$. 
$=4,26 ; D P=2,90)$ mais responsabilidades do que as que têm crianças com DT $(M=3,08 ; D P=1,97)$.

A Tabela 3 apresenta as redes sociais de apoio das famílias de ambos os grupos. Ao considerar o apoio recebido por familiares, instituições, profissionais e não familiares, constatou-se que somente no último caso há diferenças significativas. As díades com filho com DI possuem menos rede de apoio não familiar que aquelas cujos filhos têm DT, embora ambos os grupos recebam apoio frequente de familiares.

Para analisar o quanto as díades com filhos com DT e com DI dispõem de redes sociais, computou-se um total para essa variável, levando em consideração os quatro tipos de redes de apoio, isto é, familiar, não familiar, profissional e institucional. Os escores variaram entre zero - ausência total de rede de apoio - e quatro - possuir os quatro tipos de apoio. Não foram observadas diferenças significativas $\left(\mathrm{U}_{\mathrm{o}}=868,50\right.$; $p=$ $0,60)$ entre as médias das díades com filho com DT $(M$ $=3,09 ; D P=1,01)$ e com DI $(M=3,00 ; D P=0,95)$.

No tocante ao ajustamento diádico, também não foram observadas diferenças significativas ao comparar o ajustamento entre os casais dos grupos DI e DT, tanto em relação à percepção do pai quanto da mãe (Tabela 4). Ressalta-se que foram considerados não somente o total da EAD, mas também seus fatores, isto é, Consenso Diádico, Satisfação Diádica, Coesão Dádica e Expressão de Afeto. As mães com filho com DI percebem menos expressão de afeto no relacionamento com o parceiro do que aquelas que possuem filhos com DT (Tabela 5). No caso das demais medidas da EAD, não foram observadas diferenças estatisticamente significativas, isto é, pais e mães em ambos os grupos apresentam similaridades quanto ao Consenso, Satisfação e Coesão Diádica. A média geral do ajustamento é similar entre os grupos.

Ao classificar as díades como ajustadas ou desajustadas, considerando a avaliação efetuada pelos genitores, não foram observadas diferenças significativas entre os grupos DI e DT (Tabela 5). Entretanto, ao comparar os resultados percentuais de cada grupo, observa-se que, nas famílias com filho com DI, as porcentagens de "díade desajustada" são mais elevadas do que nas famílias com filho com DT.

Como as díades com filhos com DT e com DI investigadas não são homogêneas no caso da renda familiar, foram efetuadas correlações entre os escores da EAD e essa variável (Tabela 6). Só foi obtida correlação significativa para Expressão de Afeto. Essa associação foi positiva e moderada, revelando que quanto menor a renda familiar, menor a percepção de expressão de afeto nas díades com filho com DI. Ainda que outras correlações tenham sido observadas, como, por exemplo, correlação negativa entre renda e coesão para o grupo de pais cujos filhos apresentam DT, elas não foram significativas.

\section{Discussão}

O ajustamento diádico é uma medida importante que tem impacto na qualidade da relação conjugal. Os resultados deste estudo indicam que a maioria dos

Tabela 3

Discriminação da Rede Social de Apoio, Considerando os Dois Grupos (DT e DI)

\begin{tabular}{|c|c|c|c|c|c|c|}
\hline \multirow{3}{*}{ Rede social } & & \multicolumn{4}{|c|}{ Grupos } & \multirow{3}{*}{ Intergrupos } \\
\hline & & \multicolumn{2}{|c|}{$\begin{array}{l}\text { Desenvolvimento típico } \\
\text { (DT) }\end{array}$} & \multicolumn{2}{|c|}{$\begin{array}{l}\text { Deficiência intelectual } \\
\text { (DI) }\end{array}$} & \\
\hline & & $\mathrm{n}$ & $\%$ & $\mathrm{n}$ & $\%$ & \\
\hline \multirow[t]{2}{*}{ Familiar } & Não possui & 4 & 4,94 & 1 & 4,35 & ${ }^{a} 0,01$ \\
\hline & Possui & 77 & 95,06 & 22 & 95,65 & \\
\hline \multirow[t]{2}{*}{ Não Familiar } & Não possui & 13 & 16,05 & 13 & 56,52 & b15,65* \\
\hline & Possui & 68 & 83,95 & 10 & 43,48 & \\
\hline \multirow[t]{2}{*}{ Institucional } & Não possui & 22 & 27,16 & 3 & 13,04 & ${ }^{\mathrm{b}} 1,96$ \\
\hline & Possui & 59 & 72,84 & 20 & 86,96 & \\
\hline \multirow[t]{2}{*}{ Profissional } & Não possui & 35 & 43,21 & 6 & 26,09 & b2,20 \\
\hline & Possui & 46 & 56,79 & 17 & 73,91 & \\
\hline
\end{tabular}

$N=104 .{ }^{*} p<0,000 .{ }^{a} P h i .{ }^{b} \chi_{0}^{2}$.

Psico-USF, Bragança Paulista, v. 20, n. 2, p. 297-308, mai./ago. 2015 
Tabela 4

Ajustamento Diádico de Pais e Mães de Crianças com Desenvolvimento Típico (DT) e com Deficiência Intelectual (DI)

\begin{tabular}{|c|c|c|c|c|c|}
\hline \multirow{3}{*}{$\begin{array}{l}\text { Escala de } \\
\text { Ajustamento Diádico }\end{array}$} & \multicolumn{4}{|c|}{ Grupos } & \multirow{3}{*}{$\begin{array}{c}\text { Teste U de } \\
\text { Mann-Whitney }\end{array}$} \\
\hline & \multicolumn{2}{|c|}{$\begin{array}{l}\text { Desenvolvimento típico } \\
\text { (DT) }\end{array}$} & \multicolumn{2}{|c|}{$\begin{array}{c}\text { Deficiência intelectual } \\
\text { (DI) }\end{array}$} & \\
\hline & $\mathrm{M}$ & $\mathrm{DP}$ & $\mathrm{M}$ & $\mathrm{DP}$ & \\
\hline \multicolumn{6}{|l|}{ Pais } \\
\hline Consenso Diádico & 51,70 & 7,39 & 51,56 & 9,36 & 927,50 \\
\hline Satisfação Diádica & 37,24 & 5,79 & 34,02 & 7,43 & 727,50 \\
\hline Coesão Diádica & 15,26 & 3,72 & 14,78 & 3,47 & 838,00 \\
\hline Expressão de Afeto & 9,40 & 1,93 & 9,31 & 2,32 & 910,00 \\
\hline Total & 113,59 & 15,45 & 109,67 & 17,87 & 824,50 \\
\hline \multicolumn{6}{|l|}{ Mães } \\
\hline Consenso Diádico & 52,86 & 6,96 & 50,35 & 10,83 & 857,00 \\
\hline Satisfação Diádica & 36,72 & 5,94 & 33,83 & 8,37 & 767,00 \\
\hline Coesão Diádica & 15,33 & 4,08 & 15,30 & 4,82 & 917,00 \\
\hline Expressão de Afeto & 9,94 & 1,98 & 9,09 & 2,91 & $652,50 *$ \\
\hline Total & 114,85 & 14,25 & 108,57 & 20,02 & 751,50 \\
\hline
\end{tabular}

$* p<0,05$.

Tabela 5

Classificação do Ajustamento Diádico de Pais e Mães de Crianças com Desenvolvimento Típico (DT) e com Deficiência Intelectual (DI)

\begin{tabular}{|c|c|c|c|c|c|}
\hline \multirow{3}{*}{$\begin{array}{l}\text { Escala de } \\
\text { Ajustamento Diádico }\end{array}$} & \multicolumn{4}{|c|}{ Grupos } & \multirow{3}{*}{ Qui-quadrado $^{a}$} \\
\hline & \multicolumn{2}{|c|}{$\begin{array}{c}\text { Desenvolvimento } \\
\text { típico - DT }\end{array}$} & \multicolumn{2}{|c|}{$\begin{array}{c}\text { Deficiência } \\
\text { intelectual - DI }\end{array}$} & \\
\hline & $\mathrm{n}$ & $\%$ & $\mathrm{n}$ & $\%$ & \\
\hline Pais & & & & & 2,75 \\
\hline Díade desajustada & 15 & $18,52 \%$ & 8 & $34,78 \%$ & \\
\hline Díade ajustada & 66 & $81,48 \%$ & 15 & $65,22 \%$ & \\
\hline Mães & & & & & 7,53 \\
\hline Díade desajustada & 11 & $13,58 \%$ & 9 & $39,13 \%$ & \\
\hline Díade ajustada & 70 & $86,42 \%$ & 14 & $60,87 \%$ & \\
\hline
\end{tabular}

${ }^{a} N=104 ; g l=1$.

casais, de ambos os grupos, tem uma boa qualidade na relação conjugal (Consenso, Coesão, Satisfação e Expressão de Afeto), corroborando dados de estudos anteriores com casais com filhos com DI e casais com filhos sem DI, dentre os quais os de Povee et al. (2012), Wieland e Baker (2010) e Norlin e Broberg (2012). Portanto, a deficiência da criança não parece constituir um empecilho ao ajustamento do casal, conforme apontado pela literatura (Bristol et al., 1988; Carr, 1988; Da
Silva, 2011; Povee et al., 2012; Stoneman et al., 2006; Wieland \& Baker, 2010). Isso significa que ambos os grupos podem apresentar um ajustamento conjugal bom ou ruim, independentemente de se ter uma criança com deficiência intelectual na família.

O apoio/suporte marital tem sido considerado de extrema relevância na análise do ajustamento conjugal, conforme ressaltam Bristol et al. (1988), Stoneman et al. (2006), Norlin e Broberg (2012), indicando que 
Tabela 6

Correlações entre Renda Familiar e Ajustamento Diádico de Pais e Mães com Filhos com Deficiência Intelectual ou com Desenvolvimento Típico

\begin{tabular}{lcccc}
\hline & \multicolumn{3}{c}{ Grupos } \\
\cline { 2 - 5 } EAD & \multicolumn{2}{c}{ Desenvolvimento típico } & \multicolumn{2}{c}{ Deficiência intelectual } \\
\cline { 2 - 5 } & Pai & Mãe & Pai & Mãe \\
& $\mathrm{r}_{\mathrm{so}}$ & $\mathrm{r}_{\mathrm{so}}$ & $\mathrm{r}_{\mathrm{so}}$ & 0,22 \\
\hline Consenso Diádico & $-0,05$ & 0,12 & $-0,02$ & 0,27 \\
Satisfação Diádica & 0,03 & 0,15 & 0,25 & 0,22 \\
Coesão Diádica & $-0,20$ & $-0,05$ & 0,01 & $0,53^{*}$ \\
Expressão de Afeto & $-0,08$ & $-0,15$ & $0,53^{*}$ & 0,34 \\
Total & $-0,11$ & 0,10 & 0,14 & \\
\hline
\end{tabular}

${ }^{*} p<0,05$. Correlações bicaudais estatisticamente significativas para um nível de significância de 0,05.

a qualidade da relação conjugal é melhor quando há apoio do cônjuge. Embora não tenha sido encontrada diferença significativa entre os grupos, o fato de a média de ajustamento diádico das díades com filho com DI ser um pouco menor do que aquela das díades com filho com DT pode indicar uma tendência decorrente do tipo de apoio recebido pelo cônjuge que merece ser melhor investigada. De fato, mãe e pai em famílias com crianças com DI compartilharam menos a responsabilidade pelas atividades com o filho e com as tarefas domésticas. À exceção da atividade de "levar o filho para o lazer", que é a mais compartilhada com o pai, todas as demais atividades foram realizadas tendo a mãe como a principal responsável. É preciso lembrar que essa divisão menos equilibrada entre pais e mães, sendo atribuída à mãe a maior responsabilidade pelas tarefas domésticas e cuidados com os filhos, também está presente em famílias com filhos com desenvolvimento típico (Dessen \& Braz, 2000; Braz et al., 2005).

Quando a mulher assume a maior responsabilidade pelas tarefas domésticas e cuidados com os filhos, ela fica mais sobrecarregada, gerando insatisfações em diferentes dimensões da sua vida, inclusive na dimensão conjugal (Perlin \& Diniz, 2005; Perlin, 2006). A literatura tem demonstrado que as mulheres tendem a apresentar níveis mais baixos de satisfação conjugal comparados com os homens (Perlin \& Diniz, 2005). Em se tratando de diferenças entre pais e mães deste estudo, observa-se que a média de ajustamento relatado pelas mães do grupo com filho com DT é ligeiramente mais elevada do que a do pai, contrariando os dados da literatura. Esse resultado pode ser explicado pelo maior apoio recebido dos pais nesse grupo, conforme mencionado anteriormente. Já, no caso das famílias com filho com DI, os estudos indicam similaridade entre escores de pais e mães (Da Silva, 2011; Kersh, Hedvat, Hauser-Cram, \& Warfield, 2006). Na presente pesquisa, a média de ajustamento relatado pelas mães com filho com DI foi ligeiramente menor que a dos pais, o que parece compreensível, uma vez que as mães recebiam menos apoio dos pais e de instituições, além de assumirem a maior parte das responsabilidades domésticas sozinhas. No entanto, considerando não ter havido diferenças significativas, tais resultados devem ser vistos como tendências e, como tal, deveriam merecer a atenção em pesquisas brasileiras futuras. Tais tendências podem estar sinalizando a possibilidade de diferenças entre esses dois grupos, em outras amostras.

Os grupos são homogêneos quanto às características sociodemográficas, à exceção de renda e número de filhos, cujas variáveis apresentaram diferença significativa: os casais com filho com DI possuem menor renda familiar e maior número de filhos do que os casais com filho com DT. No que tange ao ajustamento diádico entre os casais, vale destacar que somente a dimensão Expressão de Afeto foi associada à variável renda familiar, tendo sido observada diferença significativa, especificamente, no caso dos casais com filho com DI. Esse fator, segundo Spanier (1976), trata da percepção subjetiva acerca da concordância ou discordância do casal em questões relativas à forma e frequência de demonstrações de carinho, afeto e desejo sexual. Assim, esse resultado indica que as mães e os pais com filho com DI percebem menos carinho de seus cônjuges, quanto menor a renda. Seria importante investigar outros fatores que podem ter implicações na demonstração de carinho dos casais em famílias de baixa renda, uma vez que a privação socioeconômica tem consequências, 
também, na saúde mental dos casais, conforme apontado por Emerson e Hatton (2009).

Em síntese, os casais com filho com DI tinham menor renda familiar, maior número de filhos e tendiam a perceber seus relacionamentos como menos ajustados. As díades com filho com DT possuíam uma maior rede de apoio do que os casais com filho com DI, que, por sua vez, contavam mais com o apoio de familiares. A rede social de apoio das famílias, que constitui uma importante variável para o bom funcionamento familiar, com implicações diretas para o relacionamento conjugal, apresentou diferenças significativas entre os grupos.

Embora as instituições desempenhem um papel importante no apoio oferecido aos casais de ambos os grupos, os casais com filho com DI contavam com uma rede limitada de apoio, quando, em realidade, eles precisariam ter uma rede mais extensa, incluindo profissionais e instituições. Além disso, pais e mães compartilhavam menos as atividades inerentes à rotina diária da família, o que pode ter influenciado a percepção de ajustamento dos casais, com as mães expressando maior insatisfação que os pais. Essa associação entre maior compartilhamento de atividades domésticas e maior contentamento, satisfação, coesão e consenso entre os casais com filhos com DI deve ser melhor investigada em pesquisas futuras.

Outro aspecto que deveria ser mais bem investigado, por sua relevância para a compreensão da dinâmica de famílias com filhos com DI, diz respeito a como os casais enfrentam os desafios cotidianos. Não há dúvidas de que esse grupo enfrenta desafios inerentes às demandas de cuidado com as necessidades especiais da criança e com a criação do filho com DI (Floyd \& Zmich, 1991), mas, de alguma maneira, os casais estão descobrindo formas variadas de lidar com esses desafios (Bailey Junior, 2007; Davis \& Gavidia-Payne, 2009), sem acarretar prejuízos severos para seu ajustamento. Conhecer tais formas de enfrentamento possibilitaria a elaboração de programas de atendimento e educação familiar mais eficazes.

É preciso ressaltar, também, que as não diferenças significativas encontradas entre os grupos quanto às dimensões de Consenso, Satisfação e Coesão sinalizam que ter um filho com DI não parece afetar o ajustamento conjugal no que concerne a tais dimensões. No entanto, conforme mencionado anteriormente, as tendências observadas nos resultados deste estudo indicam a necessidade de investigar melhor as diferenças entre esses dois grupos, em outras amostras, de preferência, utilizando uma abordagem que priorize tanto os aspectos qualitativos quanto quantitativos. Devido à complexidade dos relacionamentos familiares, a utilização de uma abordagem mista, utilizando métodos tanto qualitativos como quantitativos, tem sido adotada e destacada como sendo importante na investigação de famílias e suas relações (Clark, Huddleston-Casas, Churchill, Green, \& Garrett, 2008). Dada a escassez de estudos com casais com filho com DI, no Brasil, esta pesquisa contribui para aprofundar o conhecimento a respeito do funcionamento dessas famílias, sobretudo no que tange aos efeitos de se ter uma criança com deficiência. Os dados indicam que a deficiência do filho não constitui empecilho ao ajustamento diádico do casal, fato que deveria ser mais bem investigado em famílias brasileiras. Faz-se necessário incluir nas pesquisas um maior número de famílias, que sejam, preferencialmente, representativas das diferentes regiões geográficas, caracterizadas por uma considerável diversidade no funcionamento familiar (Torres \& Dessen, 2008). Tal inclusão permitiria compreender melhor o funcionamento de famílias de crianças que apresentam algum tipo deficiência, particularmente, no que tange ao ajustamento diádico entre casais com um filho com DI e casais com filhos com DT.

\section{Referências}

Bailey Junior, D. B. (2007). Introduction: families and disability. Mental Retardation and Developmental Disabilities Research Reviews, 13(4), 291-292.

Braz, M. P., Dessen, M. A., \& Pereira-Silva, N. L. (2005). Relações conjugais e parentais: Uma comparação entre famílias de classes sociais baixa e média. Psicologia: Reflexão e Crítica, 18(2), 151-161.

Bristol, M. M., Gallagher, J. J., \& Schopler, E. (1988). Mothers and fathers of young developmentally disabled and nondisabled boys: Adaptation and spousal support. Developmental Psychology, 24(3), 441-451.

Carr, J. (1988). Six weeks to twenty-one years old: A longitudinal study of children with Down's syndrome and their families. Journal of Child Psychology and Psychiatry and Allied Disciplines, 29(3), 407-431.

Clark, V. L. P., Huddleston-Casas, C. A., Churchill, S. L., Green, D. O. N., \& Garrett A. L. (2008). Mixed methods approaches in family science research. Journal of Family Issues, 29, 1543-1566. 
Da Silva, N. C. B. (2011). Intervenção domiciliar e envolvimento paterno: Efeitos em familias de crianças com sindrome de Down (Tese de doutorado). Centro de Educação e Ciências Humanas, Universidade Federal de São Carlos, São Carlos-SP.

Davis, K., \& Gavidia-Payne, S. (2009). The impact of child, family, and professional support characteristics on the quality of life in families of young children with disabilities. Journal of Intellectual and Developmental Disability, 34(2), 153-162.

Dessen, M. A. (2009) Questionário de caracterização do sistema familiar. In L. Weber \& M. A. Dessen (Orgs.), Pesquisando familia: Instrumentos para coleta e análise de dados (pp.102-114). Curitiba: Juruá.

Dessen, M. A., \& Braz, M. P. (2000). Rede social de apoio durante transições familiares decorrentes do nascimento de filhos. Psicologia: Teoria e Pesquisa, 16(3), 221-231.

Dessen, M. A., \& Braz, M. P. (2005). As relações maritais e sua influência nas relações parentais: implicações para o desenvolvimento da criança. In M. A. Dessen \& A. L. Costa Junior (Orgs.), A ciência do desenvolvimento humano: Tendências atuais e perspectivas futuras (pp. 132-151). Porto Alegre: Artmed.

Dykens. E. M. (2005) Happiness, well-Being, and character strengths: Outcomes for families and siblings of persons with mental retardation. Mental Retardation, 43(5), 360-364.

Emerson, E., \& Hatton, C. (2009). Socioeconomic position, poverty, and family research. In L. M. Glidden \& M. M. Seltzer (Eds.), International Review of Research in Mental Retardation: Families (pp. 97-129) New York: Elsevier.

Erel, O., \& Burman, B. (1995). Interrelatedness of marital relations and parent-child relations: $A$ meta-analytic review. Psychological Bulletin, 118(1), 108-132.

Floyd, F. J., \& Zmich, D. E. (1991). Marriage and the parenting partnership: Perceptions and interactions of parents with mentally retarded and typically developing children. Child Development, 62(6), 1434-1448.

Heyman, R., Sayers, A., \& Bellack, S. (1994). Global marital satisfaction versus marital adjustment: An empirical comparison of three measures. Journal of Family Psychology, 8(4), 32-446.
Kersh, J., Hedvat, T., Hauser-Cram, P., \& Warfield, M. E. (2006). The contribution of marital quality to the well-being of parents of children with developmental disabilities. Journal of Intellectual Disability Research, 50(12), 883-893.

Kreppner, K. (2000). The child and the family: Interdependence in developmental pathways. Psicologia: Teoria e Pesquisa, 16(1), 11-22.

Mosmann, C., Wagner, A., \& Féres-Carneiro, T. (2006). Qualidade conjugal: Mapeando conceitos. Paideia, 16(35), 315-325.

Norlin, D., \& Broberg, M. (2012). M. Parents of children with and without intellectual disability: Couple relationship and individual well-being. Journal of Intellectual Disability Research, 1-15.

Perlin, G. (2006). Casamentos contemporâneos: Um estudo sobre os impactos da interação família-trabalho na satisfação conjugal (Tese de doutorado). Universidade de Brasília, Brasília.

Perlin, G., \& Diniz, G. (2005). Casais que trabalham e são felizes: Mito ou realidade? Psicologia Clínica, 17(2), 15-29.

Povee, K., Roberts, L., Bourke, J., \& Leonard, H. (2012). Family functioning in families with a child with Down syndrome: A mixed methods approach. Journal of Intellectual Disability Research, 56(10), 961-973.

Richardson, S. S. (2012). Developmental trajectories of marriage, coparenting, and parenting stress for parents of adolescents and young adults with intellectual disability (Psychology dissertation). Georgia State University, USA. Recuperado de http://digitalarchive.gsu. edu/psych_diss/100

Risdal D., \& Singer G. H. (2004) Marital adjustment in parents of children with disabilities: A historical review and meta-analysis. Research and Practice for Persons with Severe Disabilities, 29(2), 95-103.

Scorsolini-Comin, F. \& Santos, M. A. (2010). Satisfação Conjugal: Revisão Integrativa da Literatura Científica Nacional. Psicologia: Teoria e Pesquisa, 26(3), 525-531.

Shapiro, J., Blacher, J., \& Lopez, S. R. (1998). Maternal reactions to children with mental retardation. In J. A. Burack, R. M. Hodapp \& E. E. Zigler, (Eds.) Handbook of mental retardation and development [pp. 606636]. Cambridge: Cambridge University Press. 
Spanier, G. B. (1976). Measuring dyadic adjustment: New scales for assessing the quality of marriage and similar dyads. Journal of Marriage and the Family, 38(1), 15-28.

Stoneman, Z., Gavidia-Payne, S., \& Floyd, K. (2006). Marital adjustment in families of young children with disabilities: Associations with daily hassles and problem-focused coping. American Journal on Mental Retardation, 111(1), 1-14.

Torres, C. V., \& Dessen, M. A. (2008). Brazilian culture, family, and its ethnic-cultural variety. Arizona Journal of Hispanic Cultural Studies, 12, 41 - 62.

Villas-Boas, A. C. V. B., Dessen, M. A., \& Melchiori, L. E. (2010). Conflitos conjugais e seus efeitos sobre o comportamento de crianças: Uma revisão teórica. Arquivos Brasileiros de Psicologia, 62(2), 91-102.

Wagner, A., \& Falcke, D. (2001). Satisfação conjugal e transgeracionalidade: uma revisão teórica sobre o tema. Psicologia Clínica, 13, 1-15.

Wieland, N. \& Baker, B. L. (2010). The role of marital quality and spousal support in behaviour problems of children with and without intellectual disability. Journal of Intellectual Disability Research, 54(7), 620-633.

Recebido: 04/04/2014

$1^{a}$ reformulação: 15/12/2014

$2^{a}$ reformulação: 26/12/2014

Aprovado: 05/01/2015

Sobre os autores:

Nara Liana Pereira-Silva é psicóloga, mestre e doutora em Psicologia pela Universidade de Brasília, professora do Departamento de Psicologia e do Programa de Pós-graduação em Psicologia da Universidade Federal de Juiz de Fora. Desenvolve pesquisas na área de família com membros com deficiência intelectual e tem pesquisado o estresse de genitores, a qualidade da relação conjugal, inclusão no trabalho e inclusão escolar, dentre outros temas que envolvem família e escola.

Maria Auxiliadora Dessen é psicóloga, mestre e doutora em Psicologia, possui pós-doutoramento na Universidade de Lancaster e no Instituto Max Planck para o Desenvolvimento Humano e Educação. Desde 1981, realiza pesquisas na área de Psicologia do Desenvolvimento Humano e Processos Familiares, com ênfase nas relações parentais e conjugais e suas implicações para o desenvolvimento de crianças pré-escolares e escolares.

Altemir José Gonçalves Barbosa é psicólogo, mestre em Psicologia Escolar, doutor em Psicologia, professor do Departamento de Psicologia e do Programa de Pós-graduação em Psicologia da Universidade Federal de Juiz de Fora e desenvolve pesquisas, adotando os seguintes temas: tecnologia educacional; desenvolvimento psicossocial e educação; educação inclusiva; desenvolvimento humano e novas tecnologias; informática e psicologia, dotação e talento, avaliação psicológica.

\section{Contato com os autores:}

Universidade Federal de Juiz de Fora, Instituto de Ciências Humanas, Departamento de Psicologia Rua José Lourenço Kelmer, s/n Campus Universitário. Bairro São Pedro, Juiz de Fora - Minas Gerais. CEP: 36036-900.

E-mail: naraliana.silva@ufjf.edu.br 